\title{
BPMN ALS NEUER MODELLIERUNGSSTANDARD?
}

\author{
Klaus D. Bösing
}

\section{Zusammenfassung}

Im Business Process Management (BPM) spielen Geschäftsprozessmodelle eine zentrale Rolle. Geschäftsprozessmodelle bilden die Grundlage für viele Unternehmen, um ihre Prozesse zu erfassen, zu analysieren und zu bewerten. Bezüglich der Anforderungen und Zielsetzungen gibt es unterschiedliche Modellierungsmethoden. Während in der Vergangenheit überwiegend die Methode der Ereignisgesteuerten Prozesskette (EPK) angewendet wurde, tritt heute zunehmend die Business Process Modeling and Notation (BPMN) in den Vordergrund. Im Folgenden werden beide Methoden kurz beschrieben, mögliche Transformationen dargestellt und gezeigt, welche Chancen BPMN in Zukunft hat, als Standard im Bereich der Modellierung von Geschäftsprozessen akzeptiert zu werden.

\section{Abstract}

In the business process management (BPM), business process models play a major role. Business process models describe the basic operations for many companies in order to capture, analyze and evaluate their processes. With regard to the requirements and objectives, there are different modeling methods. While the method of the event driven process chain (EPC) was predominant in the past, nowadays the business process modeling and notation (BPMN) appears increasingly in the foreground. In the following, both methods will be described briefly. Possible transformations will be described before I show what chances BPMN will have in the future to become an accepted standard in the field of modeling business processes.

\section{»I. EINLEITUNG}

Um am globalisierten Markt erfolgreich zu sein, sind beispielsweise maximale Kundenorientierung und konsequente Kostenoptimierung wichtige Voraussetzungen (Bösing 2005). Daher verfügen heute viele Unternehmen über eine Menge von Geschäftsprozessmodellen, um ihre Unternehmensziele zu erreichen. Neben der Optimierung von Geschäftsprozessen in Unternehmen dient die Modellierung von Prozessen u. a. der Dokumentation, der Automatisierung und der Simulation. Ein weiterer Grund der Modellierung besteht in der Notwendigkeit, Compliance-Anforderungen wie beispielsweise regulatorische und gesetzliche Anforderungen zu erfüllen (Strasser und Wittek 2012).

Im verstärkten Maße kommen heute Maßnahmen zur Qualitätssicherung von Geschäftsprozessen hinzu. Da Geschäftsprozessmodelle ständig verändert werden, d. h. es werden beispielsweise laufend neue Geschäftsprozesse eingefügt oder vorhandene Prozesse aktualisiert, gilt es, Methoden zu entwickeln, um die Qualität eines Prozesses innerhalb eines Geschäftsprozessmodells sicherzustellen. Da Unternehmen heute überwiegend international tätig sind, stehen zunehmend standardisierte Prozessmodelle wie CMMI, SPICE und ITIL im Vordergrund.

\section{» II. METHODEN DER MODELLIERUNG VON GESCHÄFTSPROZESSEN}

Inzwischen gibt es eine Vielzahl von Modellierungsmethoden, die sich grundsätzlich in scriptbasierte und diagrammbasierte Methoden gliedern lassen. Mit scriptbasierten Methoden erfolgt die Beschreibung mithilfe formaler Notationen in Anlehnung an Programmiersprachen. Diagrammbasierte Methoden können in datenfluss-, kontrollfluss- und objektorientierte Ansätze untergliedert werden. Neben dieser Differenzierung lassen sich die Modellierungsmethoden den fachlich-konzeptionellen und operativen Ebenen zuordnen. Die fachlichkonzeptionelle Ebene ist mehr auf der betriebswirtschaftlichen Seite angesiedelt, zu nennen sind hier beispielsweise Ereignisgesteuerte Prozessketten. Die operative Ebene tendiert in Richtung Workflowmanagement, also IT-gestützte, ausführbare Prozesse (Gadatsch 2010). Auf fachlich-konzeptioneller wie auf operativer Ebene kann ein Geschäftsprozess als eine Reihe von aufeinander folgenden Tätigkeiten oder Aktivitäten definiert werden. Ein Geschäftsprozess setzt sich aus funktions- und organisationsübergreifenden Verkettungen wertschöpfender Aktivitäten zusammen. Diese erzeugen die vom Kunden erwarteten Leistungen. Im Folgenden sollen die beiden wichtigsten Methoden, die Ereignisgesteuerte Prozesskette (EPK) und die Business Process Modeling and Notation (BPMN) vorgestellt werden.

\section{Ereignisgesteuerte Prozesskette}

A.-W. Scheer entwickelte in den 80er Jahren und publizierte in den 90er Jahren eine Modellierungsarchitektur für Geschäftsprozesse, das so genannte Architekturkonzept für Informationssysteme ARIS (Architektur Integrierter Informationssysteme). Dieses Konzept besteht aus dem Vorgehensmodell und den Modellierungsmethoden. Das ARIS-Konzept ist ein allgemeiner Bezugsrahmen für die Modellierung von Geschäftsprozessen und stellt verschiedene ebenen- und sichtenspezifische Modellierungsmethoden zur Verfügung (Scheer 2006, Slama und Nelius 2011, Staud 2006). Das ARISKonzept und das zugehörige Werkzeug sind heute im Bereich Prozessmodellierung weit verbreitet. Innerhalb der Prozess-/Steuerungssicht hat die 
EPK bzw. die erweiterte EPK (eEPK) eine zentrale Bedeutung. Mit EPKs werden Kontrollflüsse von Geschäftsprozessen in einer zeitlichen und logischen Reihenfolge abgebildet. Im Wesentlichen bestehen EPKs aus den in Abbildung 5 dargestellten Elementen.

Da die Modellierung von EPKs sehr komplex sein kann und vielfältige Modellierungsmöglichkeiten bestehen, sind definierte Modellierungsregeln unabdingbar. In vielen großen Unternehmen werden eigene ARIS-Modellierungskonventionen abgeleitet. Solche Konventionen bilden den Rahmen für einheitliche Regeln, Kriterien sowie Formate für die Modellierung. Sie sind notwendig, um konzernweit eine Vergleich- und Austauschbarkeit von Prozessmodellen zu gewährleisten. Abbildung 1 stellt ein Beispiel für ein eEPK dar.

\section{Business Process Modeling and Notation}

Die Business Process Modeling and Notation (BPMN) wurde 2004 von Stephen A. White, einem IBM-Mitarbeiter, entwickelt und von der Business Process Management Initiative (BPMI) veröffentlicht. Zielsetzung der Entwicklung von BPMN war, sowohl eine standardisierte, grafische Notation für die Modellierung von Prozessen als auch für die Prozessautomatisierung zur Verfügung zu stellen. Im Jahr 2005 übernahm die Object Management Group (OMG) die Weiterentwicklung der BPMN. 2006 wurde die BPMN in der Version 1.0 erstmals als offizieller Standard verabschiedet. Im Februar 2011 wurde die aktuell geltende Version 2.0 von der OMG veröffentlicht (Object Management Group 2011). Seitdem steht die Abkürzung BPMN für „Business Process Model and Notation“. Damit wird nicht nur die Notation, sondern auch das Business Process Definition Metamodel (BPDM) zugrunde gelegt (Freund und Rücker 2012).

BPMN-Modelle bestehen im Wesentlichen aus den in Abbildung 5 aufgeführten Elementen, die im Folgenden beschrieben werden sollen.

Slimlanes (Pools und/oder Lanes) können Systeme, Organisationseinheiten
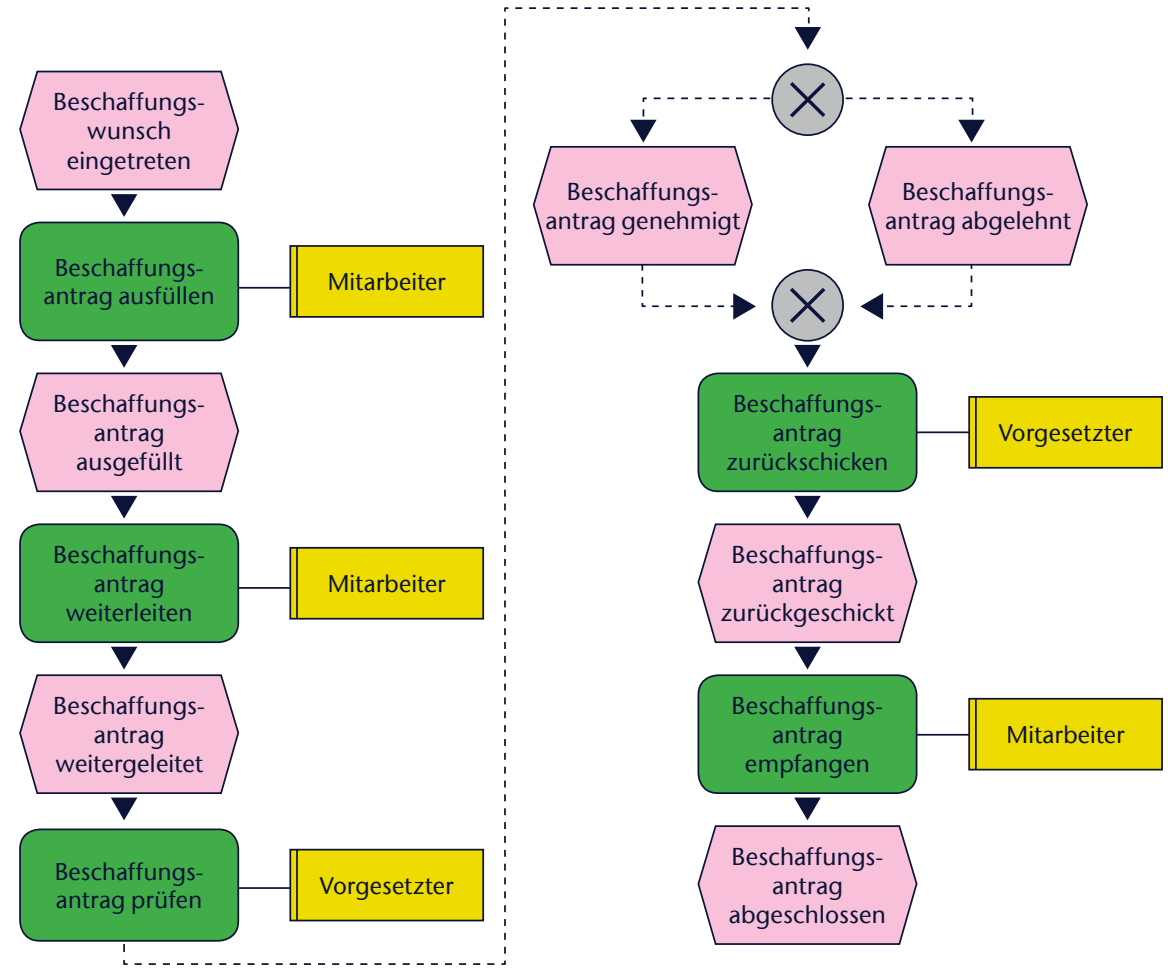

Abb. 1) Modellierungsbeispiel für ein eEPK

oder Rollen repräsentieren. In der Regel werden jedoch Organisationen als Pools und Rollen, Stellen und Organisationseinheiten als Lanes dargestellt.

BPMN differenziert zwischen Sequenzund Nachrichtenflüssen. Ein Sequenzfluss ist eine gerichtete Kante und drückt die Abhängigkeit in der Ausführung aus. Nachrichtenflüsse präsentieren die Kommunikation zwischen verschiedenen Pools.

In BPMN werden drei Arten von Flussobjekten unterschieden: Aktivität, Ereignis und Gateway. Ein zentrales Modellierungselement ist die Aktivität. Aktivitäten repräsentieren einzelne Aufgaben in einem Prozess. Diese lassen sich wiederum in atomare Task oder komplexe Unterprozesse unterteilen.

Es gibt verschiedene Formen von Ereignissen: Start-, Zwischen- und Endereignisse. Startereignisse stehen am Anfang eines Prozesses und haben keinen eingehenden Sequenzfluss. Zwischenereignisse stehen für einen Status, der sich im Verlauf einer Prozessausführung ereignet. Endereignisse stehen am Ende eines Prozesses und haben keinen ausgehenden Sequenzfluss.
Gateways werden für Verzweigungen und Zusammenführungen von Sequenzflüssen verwendet. BPMN stellt folgende Gateways zur Verfügung:

Paralleles Gateway (AND-Gateway)

Datenbasiertes inklusives Gateway (OR-Gateway)

Datenbasiertes exklusives Gateway (XOR-Gateway)

Ereignisbasiertes exklusives Gateway

Komplexes Gateway

Das parallele Gateway und die beiden datenbasierten Gateways entsprechen semantisch den Konnektoren einer EPK und sind somit hinlänglich bekannt. Das ereignisbasierte und das komplexe Gateway sollen an dieser Stelle näher betrachtet werden.

Nach einem ereignisbasierten Gateway folgen immer mehrere eintretende Ereignisse, d. h. der Sequenzfluss ist von den nachfolgenden möglichen Ereignissen abhängig. In Abbildung 2 ist der Sequenzfluss davon abhängig, ob der bestellte Artikel in den ersten zwei Wochen eingetroffen ist oder nicht. 


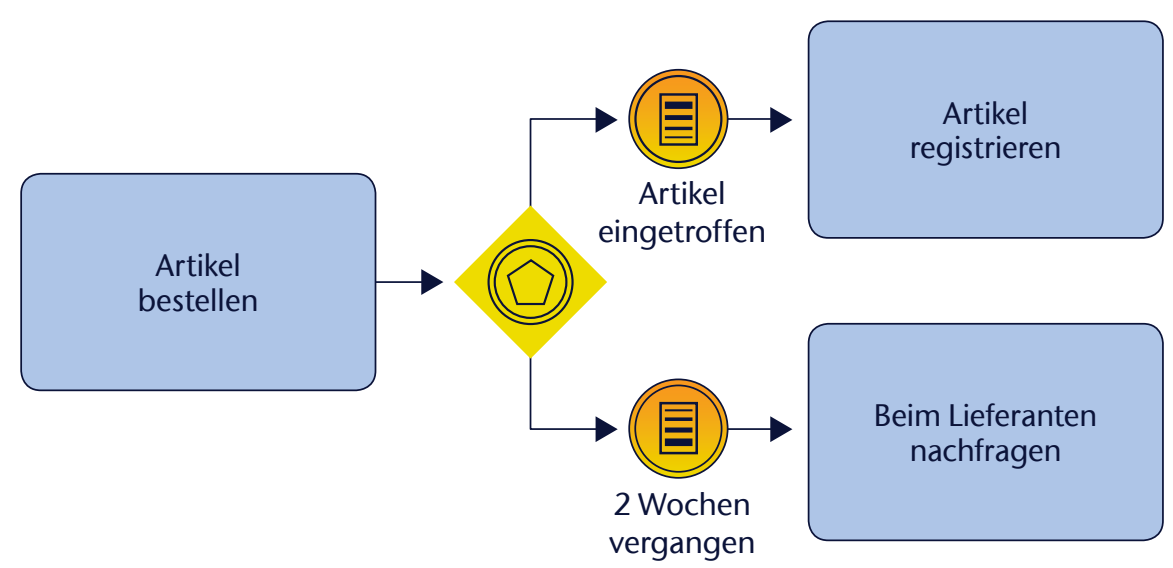

Abb. 2) Beispiel für ein ereignisbasiertes Gateway

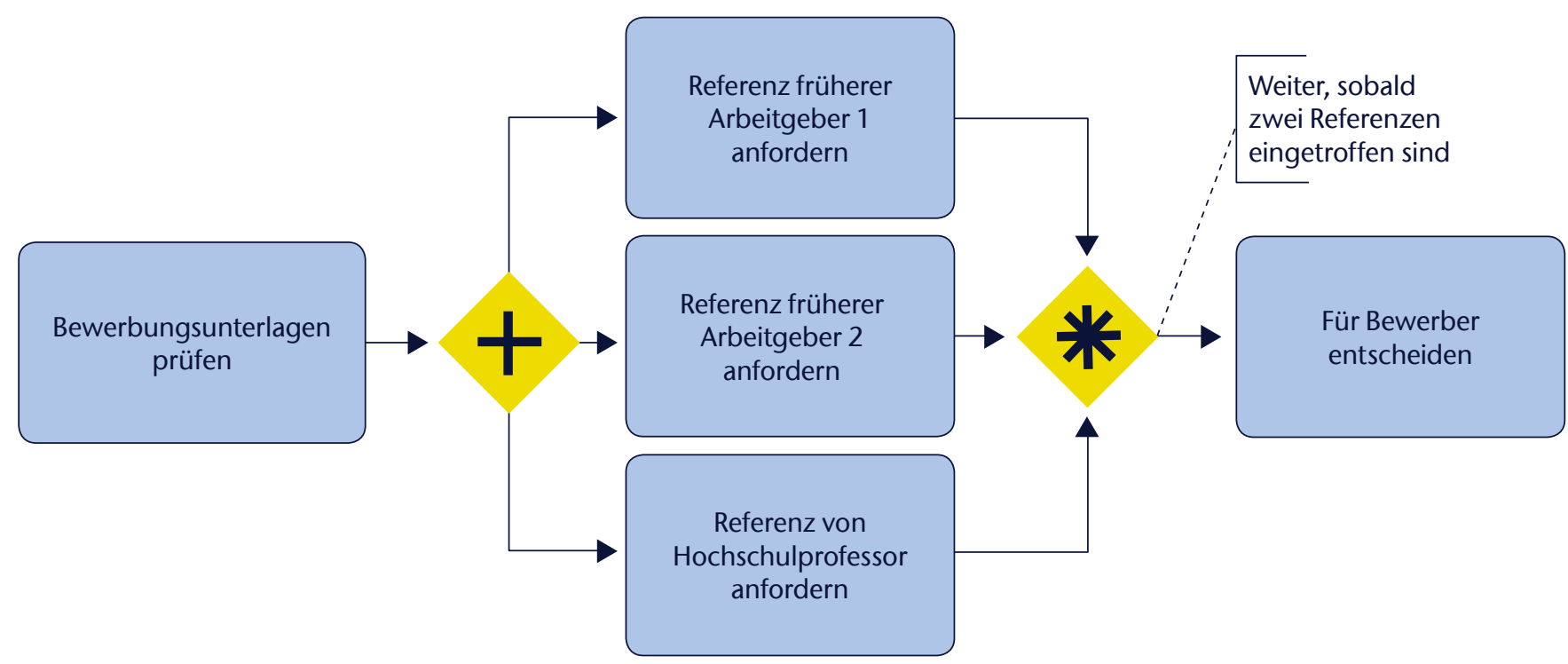

Abb. 3) Beispiel für ein komplexes Gateway (Allweyer 2009)

Es wird der Sequenzfluss fortgeführt, bei dem das nachfolgende Ereignis als erstes eingetreten ist.

Das komplexe Gateway ist ein sogenannter Alleskönner. Es wird in Szenarien angewendet, wenn sich die Semantik mit anderen Gateways nicht darstellen lässt. Die Semantik wird mithilfe von Kommentaren definiert. In Abbildung 3 wird ein Sequenzfluss eines Bewerbungsprozesses über einen parallelen Gateway in drei Sequenzflüsse gesplittet. Vorsichtshalber werden drei Referenzen angefordert, obwohl nur zwei benötigt werden, falls eine angeforderte Referenz nicht geliefert wird. Sobald zwei Referenzen eingetroffen sind, wird der Prozess fortgeführt. Die gesplitteten Sequenzflüsse werden über ein komplexes Gateway wieder zusammengeführt.

Bei der Beschreibung von Prozessen konzentriert sich BPMN im
Wesentlichen auf Sequenzflüsse, Gateways und Ereignisse. Um auch andere Aspekte zu modellieren, die für die Ausführung von Geschäftsprozessen relevant sind, werden Datenobjekte und so genannte Artefakte zur Verfügung gestellt.

Datenobjekte können Informationen, Dokumente oder Dateien sein, die erstellt, bearbeitet oder verarbeitet werden können. Sie werden über Assoziationen mit Flussobjekten verbunden, mit einer Bezeichnung versehen und darüber hinaus kann ihnen ein Status zugewiesen werden.

Zu den Artefakten gehören die Gruppierung und Annotation. Gruppierungen dienen dazu, bestehende Elemente zusammenzufassen, um sie visuell hervorzuheben. Gruppierungselemente können an beliebiger Stelle in einem Diagramm platziert werden. Annotationen können Hinweise, Bemerkungen oder Erläuterungen sein und mit einem Element über eine Assoziation verbunden werden. Gruppierungselemente und Annotationen dienen lediglich dem besseren Verständnis des Modells und haben auf die Logik keinen Einfluss. In Abbildung 4 ist noch mal das gleiche Modell dargestellt wie in Abbildung 1, allerdings mit der Modellierungsmethode BPMN.

\section{» III. EPK VS. BPMN}

Das vorrangige Ziel einer Modellierungsmethode ist es, eine entsprechende Semantik zur Verfügung zu stellen, um Geschäftsprozesse der realen Welt abzubilden. Hinsichtlich der Zielsetzung, ob das Modell beispielsweise als Grundlage für die Dokumentation, Analyse, Modifikation, Qualitätssicherung, Optimierung, Simulation oder Automatisierung von Geschäftsprozessen dient, werden unterschiedliche Anforderungen an die 


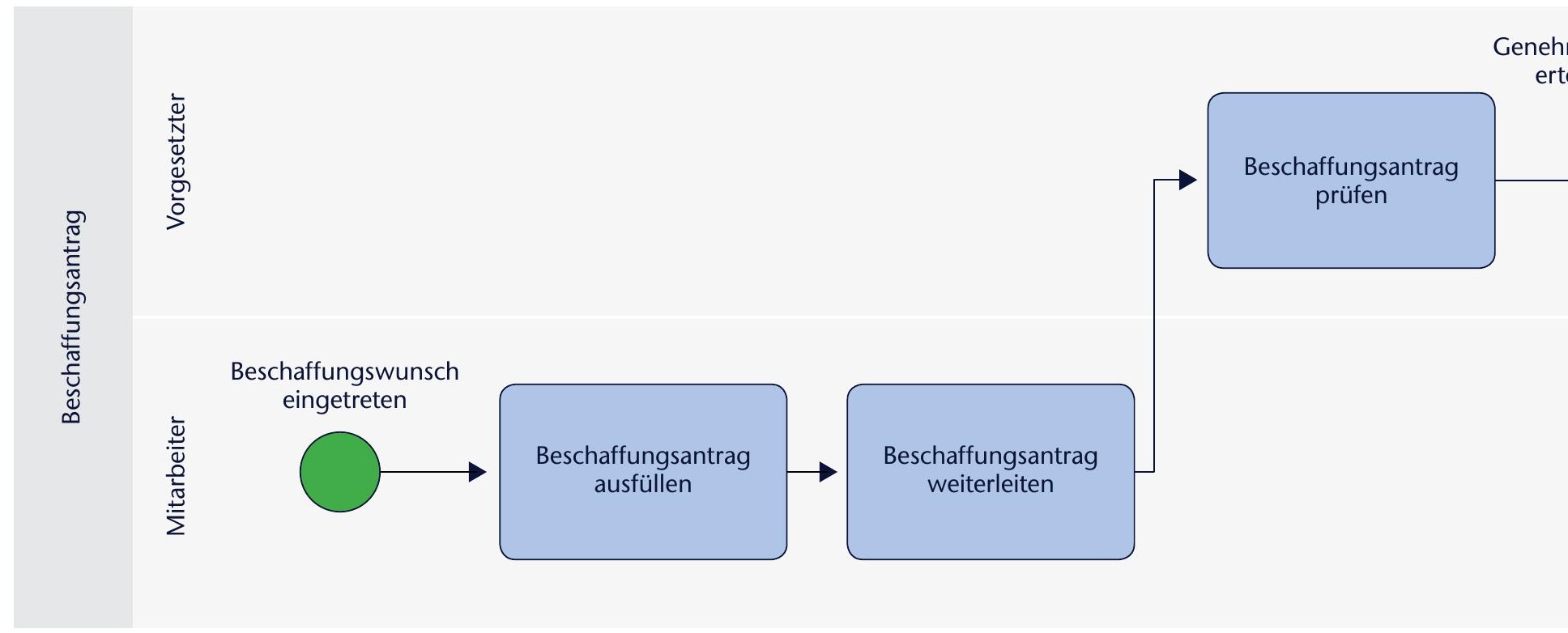

Modellierungsmethode gestellt. Wie oben schon dargestellt, lässt sich zwischen der fachlich-konzeptionellen und der operativen Ebene der Modellierung differenzieren. Für einige Anforderungen an das Prozessmodell ist sicherlich die fachlich-konzeptionelle Ebene der Modellierung völlig hinreichend, während beispielsweise hinsichtlich der Automatisierung von Geschäftsprozessen die operative Ebene eine besondere Herausforderung darstellt. Hier gilt der Anspruch, dass die Notation der Methode sowohl für die fachlich-konzeptionelle Modellierung als auch für ausführbare Geschäftsprozesse geeignet ist. Hinsichtlich der Anforderungen und der Zielsetzungen sollen die beiden vorgestellten Modellierungsmethoden näher betrachtet werden.

Zielsetzung der EPK-Methode ist es, auf der fachlich-konzeptionellen Ebene EPKs zu modellieren. Im Mittelpunkt stehen hier Kontrollflüsse, die Geschäftsprozesse in einer zeitlich und logischen Reihenfolge beschreiben. Um weitere Aspekte der Geschäftsprozessmodellierung zu berücksichtigen, lassen sich zusätzliche Elemente wie beispielsweise Rollen, Organisationseinheiten und Informationsobjekte in das Modell integrieren, die sich in einem eEPK widerspiegeln. Die EPKMethode hat sich gerade im deutschsprachigen Raum sehr etabliert und sich in vielen Unternehmen als führende Methode für die Modellierung von Geschäftsprozessen entwickelt (Gadatsch 2010). Hinzu kommt die Verbreitung der Methode durch das Modellierungswerkzeug ARIS, das auch zentraler Bestandteil des SAP-Systems ist. Nicht nur das aktuelle ARIS Business Architect \& Designer der Software AG, sondern viele andere Modellierungstools unterstützen diese Form der Modellierung. Die EPK-Methode zeichnet sich durch die einfache Beschreibung von standardisierten Abläufen wie durch die große Nähe zu Standard-Softwaresystemen aus. Hinzu kommt, dass in den letzten Jahrzehnten eine Vielzahl von Geschäftsprozess-Referenzmodellen entwickelt wurde.

Die EPK-Methode hat sich bisher nicht als formaler Standard durchsetzen können, wird aber in vielen Unternehmensbereichen als de-facto-Standard betrachtet. Neben der Problematik der Abbildung von kreativen und komplexen Geschäftsprozessen lassen sich nur bedingt Kontroll- und Überwachungsstrukturen modellieren. Aufgrund der strengen Modellierungsregel, dass eine alternierende Folge von Ereignissen und Funktionen gefordert wird, gestaltet sich eine aussagekräftige Benennung von Ereignissen häufig als problematisch. Oft haben Ereignisse keinen zusätzlichen Informationsgehalt und erscheinen redundant. Dies führt in der Praxis dazu, dass auf die Darstellung von Ereignissen oft verzichtet wird. Für die Simulation von Geschäftsprozessen ist die EPK-Methode nur eingeschränkt geeignet. Die Modellierungsmethode unterstützt keine präzise Definition von Verzweigungsbedingungen wie beispielsweise die Angabe von Wahrscheinlichkeiten. Die Modellierung von Bedingungsparametern lässt sich nur eingeschränkt umsetzen. Aufgrund des Fachkonzepts und der damit verbundenen fehlenden Workflowmodellierung ist die Überführung in ein produktives Workflow Management System (WMS) nicht möglich. In Müller 2012 wird gezeigt, dass diese Nachteile von EPKs durch die Definition geeigneter Attribute behoben werden können. Diese haben jedoch den Nachteil, dass sie eine individuelle Erweiterung und kein allgemein akzeptierter Standard sind.

Laut Spezifikation von BPMN wird das Ziel verfolgt, sowohl die Modellierung von Geschäftsprozessen als auch deren Ausführung zu berücksichtigen. Damit wird der komplette Zyklus von der Modellierung bis hin zur Implementierung realisiert. Die Notation hat den Anspruch, eine Brücke zu bilden zwischen der fachlich-konzeptionellen und der operativen Ebene, also zwischen dem Fachkonzept und der ITLösung.AufderfachlichenEbenebesteht ein wichtiger Unterschied zur EPK 

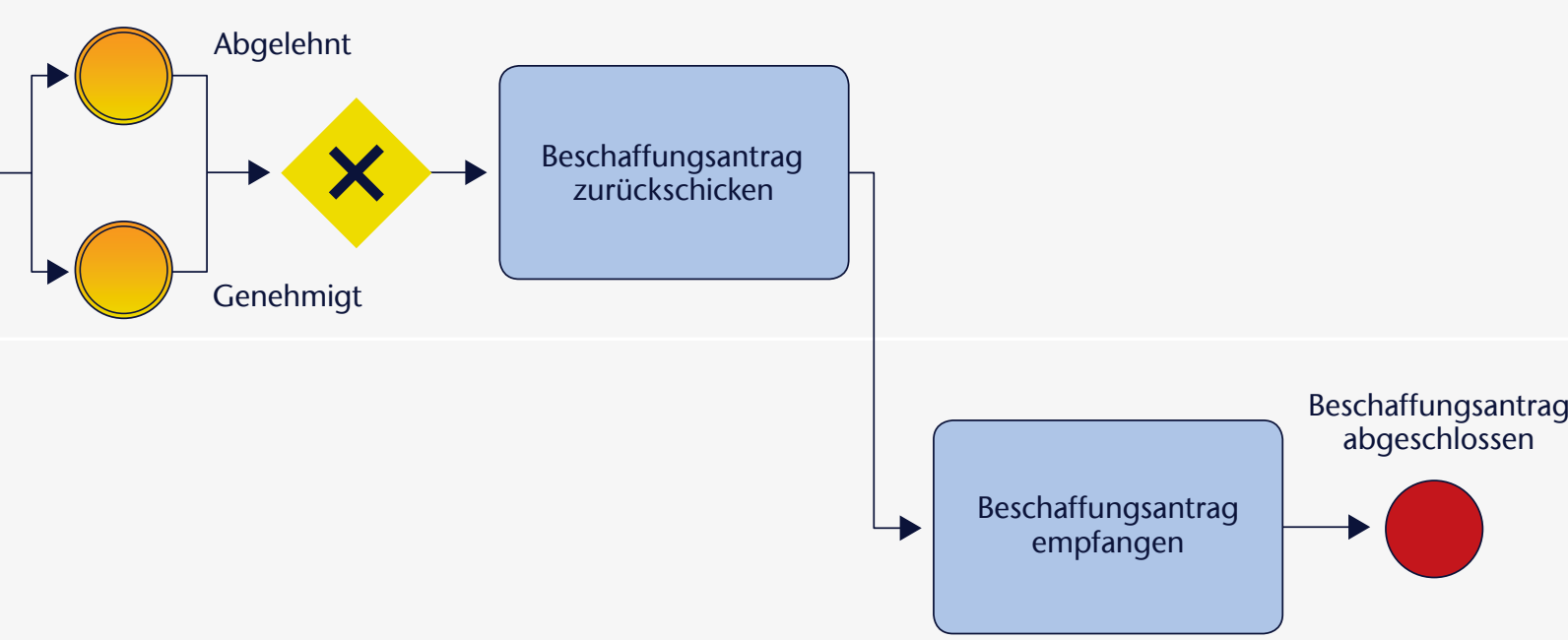

durch die klare Zuordnung von Rollen und Organisationsstrukturen mithilfe von Swimlanes. Gegenüber der EPKMethode lassen sich mit BPMN aufgrund der Notation Wartezeiten und die Dauer von Aktivitäten modellieren. In Zukunft soll es den Nutzern der BPMN möglich sein, auf der fachlichen Ebene technisch ausführbare Modelle zu entwickeln oder solche, aus denen so genannte Workflows abgeleitet werden können, die sich dann mithilfe von Process Engines eines Workflow Management Systems ausführen lassen.

BPMN entstand ursprünglich in Hinblick darauf, ausführbare Geschäftsprozesse zu modellieren, um sie mit einem WMS ausführen zu lassen. Mit dem Anspruch an die Notation, sowohl fachliche als auch technische Prozessmodelle entwickeln zu können, wird die Zukunft zeigen, wie dies in der Praxis von den Anwendern akzeptiert wird. Denn die Anforderungen an die Modellierung von fachlichen und technischen Geschäftsprozessen sind sehr unterschiedlich. Auf der fachlichen Seite steht der Prozessablauf im Vordergrund und zu viele Detailbeschreibungen wirken sich nur störend auf das Prozessmodell aus. Bei der Modellierung von technischen Prozessen sind hingegen konkrete Vorgaben notwendig, wie sich Prozesse unter welchen Bedingungen eindeutig zu verhalten haben. Hinzu kommt eine hohe Anzahl

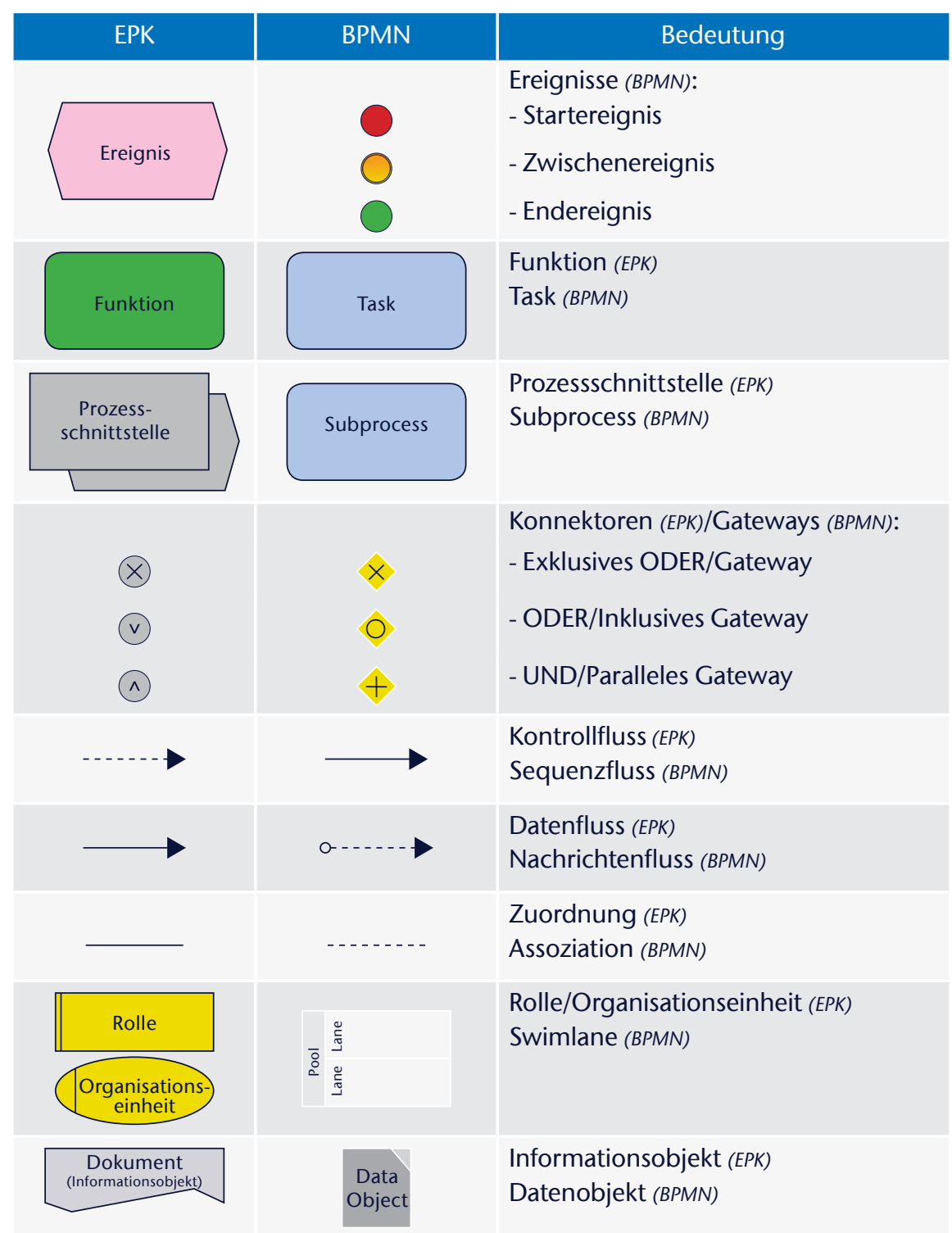

Abb. 5) Gegenüberstellung einiger EPK- und BPMN-Elemente sowie Beispiele für eine Transformation 
verschiedener grafischer Elemente, deren Eingrenzung in Zukunft notwendig sein wird, um auf fachlicher Ebene die nötige Akzeptanz zu erreichen. Durch die Übernahme der OMG und der Internationalität hat die BPMN dennoch gute Chancen, ein weltweiter Standard für die Geschäftsprozessmodellierung zu werden. Hinzu kommt die Weiterentwicklung und aktive Unterstützung namhafter Softwarehersteller wie beispielsweise SAP, Oracle und IBM.

\section{»IV. TRANSFORMATION VON EPK IN BPMN}

In vielen Unternehmen besteht heute der Wunsch, quasi auf Knopfdruck, die auf fachlicher Ebene modellierten Geschäftsprozesse als Workflow mithilfe eines Workflow Management Systems ausführen zu lassen. Viele in der Vergangenheit modellierten Geschäftsprozesse basieren auf der EPK-Methode. Es stellt sich die Frage, ob es möglich ist und mit welchem Aufwand die Überführung in den BPMNStandard verbunden ist. Prinzipiell ist eine Transformation von EPK zu BPMN durchführbar, da die Elemente der EPK und die syntaktischen Regeln eine Teilmenge von BPMN sind. Problematisch ist eine vollautomatische Transformation, da es doch zu viele Optionen gibt. Abbildung 5 zeigt eine Gegenüberstellung der grafischen Elemente.

Für die Transformation von Geschäftsprozessen von der EPK-Methode zum BPMN-Standard sind entsprechende Regeln zu definieren, so dass kein Informationsverlust entsteht. Prinzipiell sind folgende Fragen bei der Überprüfung zu klären:

Gibt es ein BPMN-Element oder eine Kombination von Elementen, welches bzw. welche die Semantik eines EPK-Elements vollständig ersetzt?

Falls kein vollwertiger Ersatz vorliegt, welches Element steht semantisch einem BPMN-Element am nächsten?

Ist die Abbildung eindeutig oder lassen sich mehrere EPK-Elemente auf ein BPMN-Element übertragen?
Wie hoch ist der Informationsverlust, wenn die Abbildung der Elemente nicht eindeutig ist?

Bedingung einer Transformation ist natürlich, dass beide Modelle vollständig konform sind, so dass kein Informationsverlust entsteht (Decker et al. 2009, Kotsev et al. 2011). Im Folgenden soll die Transformation einiger Elemente kurz dargestellt werden.

Die Funktion in einer EPK findet ihr Pendant in der Task. Da auslösende Ereignisse kurzlebige Aktivitäten innerhalb eines Prozesses repräsentieren, lässt BPMN eine weiterführende Typisierung von Aktivitäten zu. Daher können Funktionen in bestimmten Fällen mit auslösenden Zwischen- und Endereignissen transformiert werden. Ereignisse in EPKs symbolisieren einen bestimmten Zustand. Da Ereignisse keinen nennenswerten Informationsgehalt darstellen, werden sie in BPMN auch nicht berücksichtigt. Sollen trotzdem Prozesszustände modelliert werden, stehen Datenobjekte mit entsprechenden Zusatzattributen zur Verfügung. Ein interessanter Aspekt sind Startereignisse, da sie in EPKs sowohl Zustände als auch Auslöser einer Funktion, so genannte Trigger repräsentieren können. BPMN unterscheidet mehrere Typen von Triggeroptionen. Die Ermittlung des Triggertyps erfolgt durch die Beschriftung oder durch den Prozesskontext. Hat ein EPK nur ein Startereignis, so wird es in BPMN ebenfalls in nur einem Startereignis abgebildet. Sind dagegen mehrere Startereignisse vorhanden, so kann eine Transformation nur in bestimmten Fällen erfolgen. Handelt es sich um alternative Startereignisse, d. h., es folgt eine Zusammenführung der Kontrollflüsse durch einen XOR-Konnektor, kann jedes EPKStartereignis in ein BPMN-Startereignis transformiert werden. Handelt es sich nicht um alternative Startereignisse, so ist unter Berücksichtigung der Beschriftung zu prüfen, ob es mehr als einen Trigger unter den Startereignissen gibt. Ist nur ein Trigger vorhanden, wird wieder in ein BPMN-Startereignis übersetzt. Sind mehrere Trigger vorhanden, deren Kontrollflüsse durch UND- oder ODERKonnektoren zusammengeführt werden, ist eine exakte Abbildung unter Beibehaltung einer vergleichbaren syntaktischen Struktur in ein BPMN-
Modell nicht möglich. Hier ist nur eine Sequenzialisierung der parallelen Struktur denkbar. Dies hat wiederum eine Redundanz von Ereignissen zur Folge, die nicht zum besseren Verständnis des Modells beiträgt.

Konnektoren werden in BPMN mithilfe von Gateways symbolisiert. UNDKonnektoren werden in parallele Gateways, ODER-Konnektoren in datenbasierte inklusive Gateways und XORKonnektoren in datenbasierte exklusive Gateways überführt. Problematisch ist es bei ODER-Verbindungen, da hier die Semantik nicht eindeutig ist. In EPKModellen werden nach Konnektoren Verzweigungsbedingungen durch Ereignisse gekennzeichnet, während BPMN hierfür Kantenbeschriftungen notiert. Organisationseinheiten und Rollen werden in EPKs als erweiterte Informationsobjekte an Funktionen notiert, in BPMN werden sie als Pools und Lanes dargestellt. Probleme treten dann auf, wenn mehrere Organisationen und/ oder Rollen mit einer Funktion verknüpft sind. Bei der Transformation sind mehrere Lösungen möglich. In EPKs gibt es für Prozessschnittstellen zwei unterschiedliche Ausprägungen: die horizontale und vertikale Prozessschnittstelle, die im ARIS-Konzept auch als Hinterlegung bekannt ist. Die horizontale Prozessschnittstelle verbindet zwei gleichrangige Prozesse. In BPMN wird die Darstellung mittels Link-Ereignissen realisiert. Die vertikale Prozessschnittstelle entspricht einem Unterprozess in BPMN. Ob nun ein Link-Ereignis oder ein Unterprozess vorliegt, lässt sich beispielsweise klären, indem geprüft wird, ob eine Prozessschnittstelle ein Vorgängerelement und ein Nachfolgerelement aufweist. Ein Datenobjekt eines EPK-Modells wird direkt mithilfe einer gerichteten Assoziation in BPMN abgebildet.

Wie dargestellt, lassen sich viele Elemente eines EPKs in ein BPMN-Modell überführen, wenn nicht durchgehend vollautomatisch, dann aber zumindest halb automatisch. Zentrale Forderung ist natürlich, dass bei der Transformation kein Informationsverlust entsteht, so dass das Quell- und Zielmodell identisch ist. Die Überführung von EPKs nach BPMN könnte dem Wunsch vieler Unternehmen ein Stück näher kommen, 
die auf der Basis von EPKs entwickelten Geschäftsprozessmodelle als technische Prozesse ausführen zu lassen. Somit könnten die in der Vergangenheit investierten Modellierungskosten gerechtfertigt werden. So wie der BPMN-Standard Regeln für die Transformation eines Business-Modells in die Ausführungssprache Business Process Execution Language (BPEL) unterstützt, ist als alternativer Ansatz die Transformation von EPK nach BPEL in Betracht zu ziehen. (Stein und Ivanov 2007). BPEL ist eine XML-basierte Auszeichnungssprache für die Prozessautomatisierung. Grundlage des Transformationsverfahrens sind so genannte gemeinsame WorkflowPattern sowohl in EPKs als auch BPEL. Ein konkreter Ansatz wird von der Software AG mit "Model-to-Execute" verfolgt. Hier findet eine Integration von ARIS und WebMethods statt, die Anwendern eine einfache Überführung von fachlichen Prozessmodellen in technisch ausführbare Modelle erlauben soll. Für die Transformation wird BPMN 2.0 sowohl von der ARIS- als auch von der WebMethods-Komponente unterstützt (Funke 2010).

\section{» V. ZUSAMMENFASSUNG}

EPKs sind relativ einfach anzuwenden, da sie im Wesentlichen nur aus Funktionen, Ereignissen, Konnektoren und Kontrollflüssen bestehen. Die Modellierungsmethode zeichnet sich durch ihre einfache, intuitive Art und Weise aus und ist durch eine gute Interpretierbarkeit und hohe Verständlichkeit geprägt. Insbesondere die Verknüpfung von EPKs mit dem Sichtenkonzept der ARIS-Methode steuert im hohen Maße zum Verständnis bei. EPKs erfüllen grundsätzlich die fachlichen Anforderungen, die für die Geschäftsprozessmodellierung gestellt werden. Die Modellierungsmethode stößt aber aufgrund des Fachkonzepts an ihre Grenzen, da ihr die Möglichkeit der Umsetzung in automatisierte Workflows fehlt. Die Software AG stellt inzwischen gleichermaßen sowohl EPK als auch BPMN als Modellierungsmethode in ihren Softwareprodukten im Bereich BPM zur Verfügung.

Der Urgedanke der Entwicklung von BPMN war die Modellierung von
Prozessen, die mittels einer Process Engine eines Workflow Management Systems ausgeführt werden können. Inzwischen eignet sich BPMN auch für die Modellierung von Geschäftsprozessen auf fachlicher Ebene, so dass damit der Kreislauf bis hin zur IT-Lösung geschlossen ist. Die BPMN-Modellierungsmethode kommt dem Wunsch vieler Unternehmen nach, auf fachlicher Ebene „gleichzeitig“ ausführbare Prozesse zu entwickeln.

Mit BPMN wird eine Modellierungsmethode zur Verfügung gestellt, mit der umfangreiche und detaillierte Geschäftsprozessmodelle entwickelt werden können. Für die Modellierung werden inzwischen mehr als 100 Elemente mit einer ausgeprägten Semantik bereit gestellt. Aufgrund dieses Symbolumfangs besteht aber auch die Gefahr, dass in der Praxis umfangreiche und detaillierte Modelle entstehen, die nicht unbedingt zum Verständnis der Anwender beitragen. Inzwischen gibt es kritische Stimmen hinsichtlich der Benutzerfreundlichkeit von BPMN. Es werden wohl in Zukunft weiterhin entsprechend den Anforderungen und den Zielgruppen im Umfang unterschiedliche Prozessmodelle entwickelt werden. Von einem Mitglied der OMG wird gar eine Unterteilung der Notationselemente in vier Klassen vorgeschlagen, von "simple“ mit nur sieben Elementen bis hin zur Klasse "complete“ (Shapiro 2010).

Aufgrund der Übernahme durch die OMG als wichtige Institution im ITBereich und durch weltweit agierende Standardisierungsgremien, hat BPMN dennoch große Chancen, als Standard im Bereich der Geschäftsprozessmodellierung akzeptiert zu werden. Hinzu kommt eine agile Community der BPMN-Modellierung sowie die Existenz von über 70 Herstellern von Modellierungstools, die diese Methode inzwischen unterstützen.
LITERATUR

Allweyer, T. (2009): BPMN 2.0 - Business Process Model and Notation, 2. Auflage, Books on Demand, Norderstedt.

Bösing, K. D. (2005): Methodologie zur Optimierung von Geschäftsprozessen. In: Wissenschaftliche Beiträge der TH Wildau [FH], 19-26, Wildau.

Decker, G., Tscheschner, W., Puchan, J. (2009): Migration von EPK zu BPMN. In: EPK 2009, Geschäftsprozessmanagement mit Ereignisgesteuerten Prozessketten, Proceeding, 91-109, Nüttgens, M. Rump, F. J., Mendling J., Gehrke, N. (Hrsg.), 8. Workshop der Gesellschaft für Informatik e.V., Berlin.

Funke, S. (2010): Software AG komplettiert ihre Prozessplattform, Computerwoche, 10.12.2010, http://www. computerwoche.de/software/software-infrastruktur/ 2359954, Zugriff: 10.09.2012.

Gadatsch, A. (2010): Grundkurs GeschäftsprozessManagement, 6. Auflage, Vieweg+Teubner Verlag, Wiesbaden.

Freund, J., Rücker, B. (2012): Praxishandbuch BPMN 2.0, 3. Auflage, Carl Hanser Verlag, München.

Kotsev, V., Stanev, I, Grigorova, K. (2011): BPMN-EPCBPMN Converter. In: Imagination, Creativity, Design, Development, Proceeding of the International Students Conference on Informatics, ICDD, 150-156, Simian, D. (Ed.), Sibiu, Romania.

Müller, Chr. (2012): Generation of EPC based Simulation Models. In: ECMS 2012, Proceedings, 301-305, 26. European Conference on Modelling and Simulation, Troitzsch, K. G.; Möhring M.; Lotzmann, U. (Eds.), Universität Koblenz-Landau, Koblenz.

Object Management Group (2011): Business Process Mo del and Notation (BPMN) Version 2.0, http://www.omg org/spec/BPMN/2.0, Zugriff: 08.09.2012.

Scheer, A.-W. (2006): ARIS - Vom Geschäftsprozess zum Anwendungssystem, 4. Auflage, Springer-Verlag, Berlin.

Shapiro, R. (2010): Update on BPMN Release 2.0, February 3, 2010, http://www.wfmc.org/download-document/ bpmn-2.0-industry-update-presentation.html, Zugriff: 10.09.2012

Slama, D., Nelius, R. (2011): Enterprise BPM, 1. Auflage, dpunkt.verlag, Heidelberg.

Staud, J. (2006): Geschäftsprozessanalyse: Ereignisorientierte Prozessketten und objektorientierte Geschäftsprozessmodellierung für Betriebswirtschaftliche Standard Software, 3. Auflage, Springer-Verlag, Berlin.

Stein, S., Ivanov, K. (2007): EPK nach BPEL Transformation als Voraussetzung für praktische Umsetzung einer SOA. In: Software Engineering 2007, 75-80, Böttinger, S. Theuvsen, S. L. Rank, S. Morgenstern, M. (Eds.), Fachtagung des GI-Fachbereichs Softwaretechnik, Hamburg.

Strasser, A., Wittek, M. (2012): IT-Compliance, Informatik Spektrum, Band 25, Heft 1, Februar 2012, 39-44, Springer-Verlag, Berlin.

\section{AUTOR}

Prof. Dr. Klaus D. Bösing

Fachbereich Betriebswirtschaft/Wirtschaftsinformatik Fachgebiet Software Engineering

TH Wildau [FH]

Telefon: +49 (0) 3375 / 508-952

klaus.boesing@th-wildau.de 